\title{
Optimization model and algorithm for rolling stock maintenance scheduling in metro
}

\author{
L. Ma ${ }^{1}$, X.-X. Zhang ${ }^{1}$, J. Guo ${ }^{2} \&$ X.-M. Wang ${ }^{2}$ \\ ${ }^{1}$ School of Optoelectronic Information, \\ University of Electronic Science and Technology of China, China \\ ${ }^{2}$ School of Information Science and Technology, \\ Southwest Jiaotong University, China
}

\begin{abstract}
The efficiency of metro rolling stock maintenance directly affects the safety and capability of train operation in the main line. Aiming at the optimization problem of rolling stock maintenance scheduling (RSMS) in metro, a multi-objective mixed integer nonlinear bi-level programming model was presented in this paper to improve the efficiency of maintenance as well as the availability of the rolling stock in the planning horizon. In the model, maintenance systems, train operation timetable in the main line and maintenance capacity of the crews are taken into comprehensive consideration. To solve the optimal solutions quickly, the static variable ordering heuristics according to a possible minimum value of the start time of the maintenance, the dynamic value ordering heuristics according to the minimum value and the constraint propagation based on the maintenance cycle are imbedded in the basic backtracking algorithm. Finally, the effectiveness of the model and the algorithm is verified by the instances from Chengdu Metro Line 2 in China.

Keywords: metro, rolling stock maintenance scheduling, bi-level programming, improved backtracking algorithm.
\end{abstract}

\section{Introduction}

Every rolling stock must undergo maintenance checks after a certain number of hours or kilometres of travelling time or distance. Most of the maintenances are detailed in the metro rolling stock bases including car depot and parking lot. Maintenance procedures can be divided into preventive maintenance and 
corrective maintenance depending on their level. Compared with preventive maintenance, corrective maintenance has greater randomness. At the same time, daily inspection, one kind of the preventive maintenance, must be carried out every day. The other preventive maintenance procedures are divided depending on their level. In order to reduce the maintenance costs and to extend the rolling stock life, RSMS is established to arrange a variety of maintenances coordinately.

In current practice, the decision making process for RSMS is largely manual and primarily relies on the knowledge and judgment of experts, which leads to low efficiency and error-prone. Therefore, it is essential to study the optimization theory of RSMS. Some metro rolling stock maintenance information systems were built to assist the scheduler to dispatch the daily maintenances in [1-3]. But the systems are only limited to add, delete and change the maintenances in [1, 2]. A 0-1 programming model and a modified genetic algorithm were established to maximize the matching between the maintenance workers and the maintenance works in [3]. But the skill indicators of the crews are non-deterministic and changing, furthermore, because of the great flexibility of the actual maintenance, it is difficult to divided one maintenance task into several processes. The railway routing considering maintenance problem the track maintenance production team scheduling problem are discussed in [4] and [5], but not actual RSMS optimization problem.

In this paper, a multi-objective mixed integer nonlinear bi-level programming model for RSMS is established. In the model, metro RSMS is to determine the daily maintenance content, time and crews of each rolling stock, meanwhile the sum of the present maintenances and the availability of the rolling stocks are maximized. Then, an improved backtracking algorithm is designed to solve the model and achieve different kinds of maintenances for a planning horizon. Finally, experimental results based on the instances from Chengdu Metro Line 2 in China show that the model and algorithm could be a promising way.

\section{A mixed integer nonlinear bi-level programming model}

\subsection{Assumptions}

In order to model, assumptions are as follows:

(1) Maintenance systems, daily train operation schedule in the main line, daily capacity of the maintenance crew teams and the time when each rolling stock is put into operation are determined and unchanged.

(2) Neither maintenance can be interrupted until the end.

(3) One maintenance requires only one maintenance crew team from the start to the end, while one maintenance crew team can only undertake one maintenance at one time.

(4) There is only one rolling stock base, that is, only one car depot and excluding subsidiary parking lot, which indicates it doesn't need to allocate place for each maintenance. 


\subsection{Constants}

Based on the above assumptions constants are defined as follows:

(1) $T=\left\{t_{1}, \cdots, t_{k}, \cdots, t_{N^{T}}\right\}$ represents the maintenance scheduling horizon taking one day as a step. $t_{1}$ is the start time, $t_{N^{T}}$ is the end time. $g_{k}$ is the number of submitting rolling stocks to the main line when $t_{k}$, while $a_{k}$ is the total capacity of the maintenance crew teams when $t_{k}$.

(2) $R=\left\{r_{1}, \cdots r_{j}, \cdots, r_{N^{R}}\right\}$ is formed by different kinds of maintenances sorted by the level from low to high. $r_{j}=\left\{y_{j}, p_{j}, T_{j}^{\min }, T_{j}^{\max }, d_{j}\right\}$ is the $j$-th kind of the maintenance, $y_{j}$ is the designation, $p_{j}$ is the priority, $p_{j_{1}}>p_{j_{2}}$ indicates the level of $r_{j_{1}}$ is higher than the $r_{j_{2}} \cdot T_{j}^{\min }$ and $T_{j}^{\max }$ are the shortest and longest maintenance cycle. $d_{j}$ is the duration time of $r_{j}$, , generally, the higher the maintenance level, the longer the consuming time.

(3) $C=\left\{c_{1}, \cdots c_{i}, \cdots, c_{N^{c}}\right\}$ is composed of $N^{C}$ rolling stocks. $t_{i, j}^{0}$ is the latest end time of maintenance $r_{j}$ of rolling stock $c_{i}$ before $t_{1}$, while $t_{i}^{s}=\max \left(t_{i, j}^{0}\right)$ is the latest end time of all maintenances of rolling stock $c_{i}$ before $t_{1}$.

\subsection{Variables}

On condition that $t_{i, j}^{0}$ is known, the domains of start time and end time of the first maintenance $r_{j}$ of rolling stock $c_{i}$ can be expressed as:

$$
\begin{gathered}
T_{i, j, 1}^{s}=\left[t_{i, j}^{0}+T_{j}^{\min }+1, t_{i, j}^{0}+T_{j}^{\max }+1\right] \\
T_{i, j, 1}^{e}=T_{i, j, 1}^{s}+d_{j}-1=\left[t_{i, j}^{0}+T_{j}^{\min }+d_{j}, t_{i, j}^{0}+T_{j}^{\max }+d_{j}\right]
\end{gathered}
$$

Make $T_{i, j, 0}^{e}=t_{i, j}^{0}$, the domains of start time and end time of the $l$-th $(l \geq 1)$ maintenance $r_{j}$ can be expressed as:

$$
\begin{gathered}
T_{i, j, l}^{s}=\left[t_{i, j}^{0}+l \times T_{j}^{\min }+(l-1) \times d_{j}+1, t_{i, j}^{0}+l \times T_{j}^{\max }+(l-1) \times d_{j}+1\right], \\
T_{i, j, l}^{e}=\left[t_{i, j}^{0}+l \times T_{j}^{\min }+l \times d_{j}, t_{i, j}^{0}+l \times T_{j}^{\max }+l \times d_{j}\right]
\end{gathered}
$$

As long as any maintenance starts in $T$, we can think the maintenance belongs to the scheduling, then the minimum number and the maximum number of $r_{j}$ within $T$ are expressed as:

$$
N_{i, j}^{\min }=\frac{t_{N^{T}}-t_{i, j}^{0}-1+d_{j}}{T_{j}^{\max }}, N_{i, j}^{\max }=\frac{t_{N^{T}}-t_{i, j}^{0}-1+d_{j}}{T_{j}^{\min }}
$$


Then, the minimum maintenance number and the maintenance maximum number of $c_{i}$ in $T$ are expressed as:

$$
N_{i}^{\min }=\sum_{j=1}^{N^{R}} N_{i, j}^{\min }, N_{i}^{\max }=\sum_{j=1}^{N^{R}} N_{i, j}^{\max }
$$

Make the duration of $r_{j}$ be expressed as:

$$
D_{i, j, l}=T_{i, j, l}^{s} \cup T_{i, j, l}^{e}
$$

Then, the maximum domain of all $r_{j}$ and the maximum domain of all kinds of maintenances of $c_{i}$ in $T$ are expressed as:

$$
T_{i, j}=\bigcup_{l=1}^{N_{i, j}^{\max }} D_{i, j, l}, \tilde{T}=\bigcup_{i=1}^{N^{C}} \bigcup_{j=1}^{N^{R}} T_{i, j}
$$

Hence, the optional variable $o_{i, j, l}\left(1 \leq i \leq N^{C}, 1 \leq j \leq N^{R}, 1 \leq l \leq N_{i, j}^{\max }\right)$ is defined to denote whether $c_{i}$ needs to execute the $l$-th $r_{j} . t_{i, j, l}^{e}$ can be determined as long as the determination of $t_{i, j, l}^{s}$, therefore, the variable $t_{i, j, l}^{s} \in T_{i, j, l}^{s}$ indicates start time of the $l$-th $r_{j}$ when $o_{i, j, l}=1$.

According to $o_{i, j, l}$, the variable $v_{i, j, k} \in\{0,1\}$ is defined to represent whether the maintenance of $c_{i}$ is $r_{j}$ at $t_{k}\left(t_{k} \in \tilde{T}\right)$. Namely, if $o_{i, j, l}=1$, then $v_{i, j, k}=1$ and $t_{k} \in\left[t_{i, j, l}^{s}, t_{i, j, l}^{e}\right]$, otherwise, $\quad v_{i, j, k}=0 \quad$ and $t_{k} \in T_{i, j, l}^{s} \cup T_{i, j, l}^{e}$. If $N_{i, j}^{\max }>1$, the difference between the start time of the $l$-th $r_{j}$ of $c_{i}$ and the end time of $l$-1-th $r_{j}$ of $c_{i}$ can be expressed as $d_{i, j, l}$, which is equal to $t_{i, j, l}^{s} \times o_{i, j, l}-t_{i, j, l-1}^{e} \times o_{i, j, l-1}-1$. If $l=1$, then $d_{i, j, l}=t_{i, j, l}^{s} \times o_{i, j, l}-t_{i, j}^{0}-1$.

\subsection{Objective functions}

Because of maintenance cycles, daily capacity of the maintenance crews, etc., not every maintenance can be executed. Thus, the main purpose of the rolling stock maintenance scheduling is to arrange the maintenances as many as possible. The main objective is expressed as formula (7).

$$
\max f_{1}=\sum_{i=1}^{N^{C}} \sum_{j=1}^{N^{R}} \sum_{l=1}^{N_{i, j}^{\max }} o_{i, j, l}
$$

In order to improve rolling stock turnover rate and delivery capacity of the rolling stock to the main line, the secondary objective is defined to maximize the availability of the rolling stocks. 


$$
\min f_{2}=\frac{\sum_{i=1}^{N^{C}} \sum_{j=1}^{N^{R}} \sum_{l=1}^{N_{i, j}^{\max }}\left(o_{i, j, l} \times d_{j}\right)}{\sum_{i=1}^{N^{C}} \sum_{j=1}^{N^{R}} \sum_{l=1}^{N_{i, j}^{\max }} o_{i, j, l}}
$$

\subsection{Constraints}

(1) There is only one maintenance for any rolling stock at any time, that is, the uniqueness constraint $b_{1}$ is expressed as:

$$
\sum_{1 \leq j \leq N_{i, j}^{\max }, t_{i, j, l}^{s} \leq t_{k} \leq t_{i, j, l}^{e}} v_{i, j, k} \leq 1, i=1,2, \ldots, N^{C}, l=1,2, \ldots, N_{i, j}^{\max }, t_{k} \in \tilde{T}
$$

(2) The maintenance cycle constraint $b_{2}$ between the adjacent maintenances with the same kind can be expressed as:

$$
\begin{gathered}
T_{j}^{\min } \leq d_{i, j, l} \leq T_{j}^{\max }, \\
i=1,2, \ldots, N^{C}, j=1,2, \ldots, N^{R}, l=1,2, \ldots, N_{i, j}^{\max }
\end{gathered}
$$

(3) If one low-level maintenance is after another high-level maintenance, then the difference between them must be less than maintenance cycle of the low-level maintenance. And maintenance cycle constraint $b_{3}$ can be described below.

$$
\begin{gathered}
T_{j_{1}}^{\min } \leq t_{i, j_{1}, l}^{s}-t_{i, j_{2}, l-1}^{e}-1 \leq T_{j_{1}}^{\max } \\
i=1,2, \ldots, N^{C}, j_{1}, j_{2}=1,2, \ldots, N^{R}, p_{j_{1}}<p_{j_{2}}, l=2,3, \ldots, N_{i, j}^{\max }
\end{gathered}
$$

(4) Each maintenance can't be interrupted until the end, and uninterruptible constraint $b_{4}$ is described as follows.

$$
\begin{gathered}
v_{i, j_{1}, k_{1}} \times y_{j_{1}}=v_{i, j_{2}, k_{2}} \times y_{j_{2}}, i=1,2, \ldots, N^{C}, \\
j_{1}, j_{2}=1,2, \ldots, N^{R}, t_{k_{1}}, t_{k_{2}} \in\left[t_{i, j_{1}, l}^{s}, t_{i, j_{1}, l}^{e}\right], l=1,2, \ldots, N_{i}^{\max }
\end{gathered}
$$

(5) If one low-level maintenance and one high-level maintenance both can be assigned to some rolling stock at some time, then the high-level maintenance must be scheduled. Hence, the covering constraint $b_{5}$ is expressed as follows.

$$
\begin{gathered}
O_{i, j_{1}, l_{1}}>O_{i, j_{2}, l_{2}}, \\
i=1,2, \ldots, N^{C}, j_{1}, j_{2}=1,2, \ldots, N^{R}, p_{j_{1}}>p_{j_{2}}, T_{i, j_{1}, l_{1}}^{s} \cap T_{i, j_{2}, l_{2}}^{s} \neq \varnothing
\end{gathered}
$$

(6) The daily capacity of the maintenance crews is limited, as a result, the total number of the maintenances scheduled every day is restricted. The maintenance capacity constraint $b_{6}$ is expressed as follows. 


$$
\sum_{1 \leq i \leq N^{c}} \sum_{1 \leq j \leq N_{i, j}^{\max }, t_{i, j, l}^{i} \leq t_{k} \leq t_{i, j, l}^{e}} o_{i, j, l} \leq a_{k}, t_{k} \in \tilde{T}
$$

(7) In order to meet the operational requirements of daily train timetable, a certain amount of rolling stock must be submitted to run in the main line, that is, train operation constraint in the main line $b_{7}$ can be described as follows.

$$
N^{C}-\sum_{1 \leq i \leq N^{C}} \sum_{1 \leq j \leq N_{i, j}^{\max }, t_{i, j, l}^{e} \leq t_{k} \leq t_{i, j, l}^{e}} o_{i, j, l} \geq g_{k}, t_{k} \in \tilde{T}
$$

\subsection{Bi-level programming model}

The above two objective functions are in conflict, at the same time, it is meaningful that the objective function (8) is optimized after the objective function (7) has been optimized. Therefore, the model can be transformed into a smooth nonlinear bilevel programming model $[6,7]$.

Let $o_{i, j, l}, \quad t_{i, j, l}^{s}$ and $t_{i, j, l}^{e}$ form a generalized variable donated as $x_{i, j, l}=<o_{i, j, l}, t_{i, j, l}^{s}, t_{i, j, l}^{e}>$. . Let $b_{m}\left(x_{i, j, l}\right)=1$, if $x_{i, j, l}$ satisfies the constraint $b_{m}(m=1, \ldots, 7)$, , otherwise, $b_{m}\left(x_{i, j, l}\right)=0$. Then the feasible solution set in the whole optimization model is denoted as follows.

$$
S\left(x_{i, j, l}\right)=\left\{x_{i, j, l} \mid b_{m}\left(x_{i, j, l}\right)=1,1 \leq i \leq N^{C}, 1 \leq j \leq N^{R}, 1 \leq l \leq N_{i, j}^{\max }\right\}
$$

And the optimal solution set of the upper level programming model is denoted as follows.

$$
\psi_{1}\left(x_{i, j, l}\right)=\arg \max _{x_{i, j, l}}\left\{f_{1}\left(x_{i, j, l}\right): x_{i, j, l} \in S\right\}
$$

If $\psi_{1}\left(x_{i, j, l}\right) \neq \varnothing$, let the optimal value of the upper objective function (7) be denoted as $f_{1}^{o p t}$, then the optimal solution set of the lower level programming model is denoted as follows.

$$
\psi_{2}\left(x_{i, j, l}\right)=\arg \min _{o_{i, j, k}}\left\{f_{2}\left(x_{i, j, l}\right): x_{i, j, l} \in S, x_{i, j, l} \in \psi_{1}, f_{1}\left(x_{i, j, l}\right) \geq f_{1}^{o p t}\right\}
$$

\section{Improved backtracking algorithm}

In order to improve the efficiency of the basic backtracking algorithm [8], the static variable ordering heuristics (VarOH) $[9,10]$ according to a possible minimum value of the start time of the maintenance and the dynamic value ordering heuristics $(\mathrm{ValOH})[11,12]$ according to the minimum value are designed in this improved backtracking algorithm. Furthermore, the constraint propagation (CPr) [12] based on the maintenance cycle were imbedded to communicate the domain reduction of a variable to all of the constraints that are stated over this variable. 


\subsection{Static variable ordering heuristics}

The maintenances number of the rolling stock $c_{i}$ is most up to $N_{i}^{\max }$ in the planning horizon $T$, and sort these generalized variables $x_{i, j, l}=<o_{i, j, l}, t_{i, j, l}^{s}>$ by the possible minimum value $t_{i, j}^{0}+l \times T_{j}^{\min }+l \times d_{j}$ of the start time $t_{i, j, l}^{s}$. If there are multiple value domains of the start time overlap, then schedule the high-level maintenances in front of the low-level maintenances. Rank the variables among the different rolling stocks according to the existing order, hence, the reordering of variables is denoted as $X=\left\{X_{1}, \ldots, X_{i}, \ldots, X_{N^{c}}\right\}$, and $X_{i}=\left\{x_{i, n_{i}} \mid 1 \leq n_{i} \leq N_{i}^{\max }\right\} . \quad x_{i, n_{i}}=<o_{i, n_{i}}, t_{i, n_{i}}^{s}>$ represents the $n_{i}$-th generalized variable, $o_{i, n_{i}}$ and $t_{i, n_{i}}^{s}$ indicate the optional variable and the start time variable of the $n_{i}$-th maintenance of the rolling stock $c_{i}$ respectively.

\subsection{Dynamic value ordering heuristics}

In order to make the algorithm search better solution as soon as possible, it is needed to prioritize the values in the domain of each variable. Let the priority value of $x_{i, n_{i}}=<o_{i, n_{i}}, t_{i, n_{i}}^{s}>$ be $<1, \min \left(T_{i, n_{i}}^{s}\right)>$, and $T_{i, n_{i}}^{s}$ is the value domain of the start time variable $t_{i, n_{i}}^{s}$.

\subsection{Constraint propagation}

CPr [12] contains domain reduction and communicating, which is a process of communicating the domain reduction of a variable to all of the constraints that are stated over this variable. Based on the maintenance cycle constraints (see formula (10) and formula (11)) and the relationships between the start time and the end time of the contiguous maintenances (see formula (2)), and if the start time and the end time of $(l-1)$-th maintenance $r_{j}$ are determined, then the domain of start time of $l$-th maintenance $r_{j}$ can be narrowed as follows.

$$
T_{i, j, l}^{s}=\left[t_{i, j, l-1}^{e}+T_{j}^{\min }+1, t_{i, j, l-1}^{e}+T_{j}^{\max }+1\right]
$$

Then, according to the following communicating formula.

$$
T_{i, j, l+1}^{s}=\left[T_{i, j, l}^{e}+T_{j}^{\min }+1, T_{i, j, l}^{e}+T_{j}^{\max }+1\right]
$$

Finally, reduce the value domain of the start time of larger than $l$-th maintenance.

\subsection{Algorithm steps}

Let that the optimal solution set be denoted by $\psi_{1}$, the initial minimum value of the objective function ( 7$)$ be 0 , the constraint set of all constraints $b_{m}(m=1, \ldots, 7)$ be denoted as $B$, invoking constraint propagation method be described as $\operatorname{Cpr}(B)$, 
then the steps of the improved backtracking algorithm (Improved-BT) is used to solve the upper level model with the objective function (7) are designed as follows.

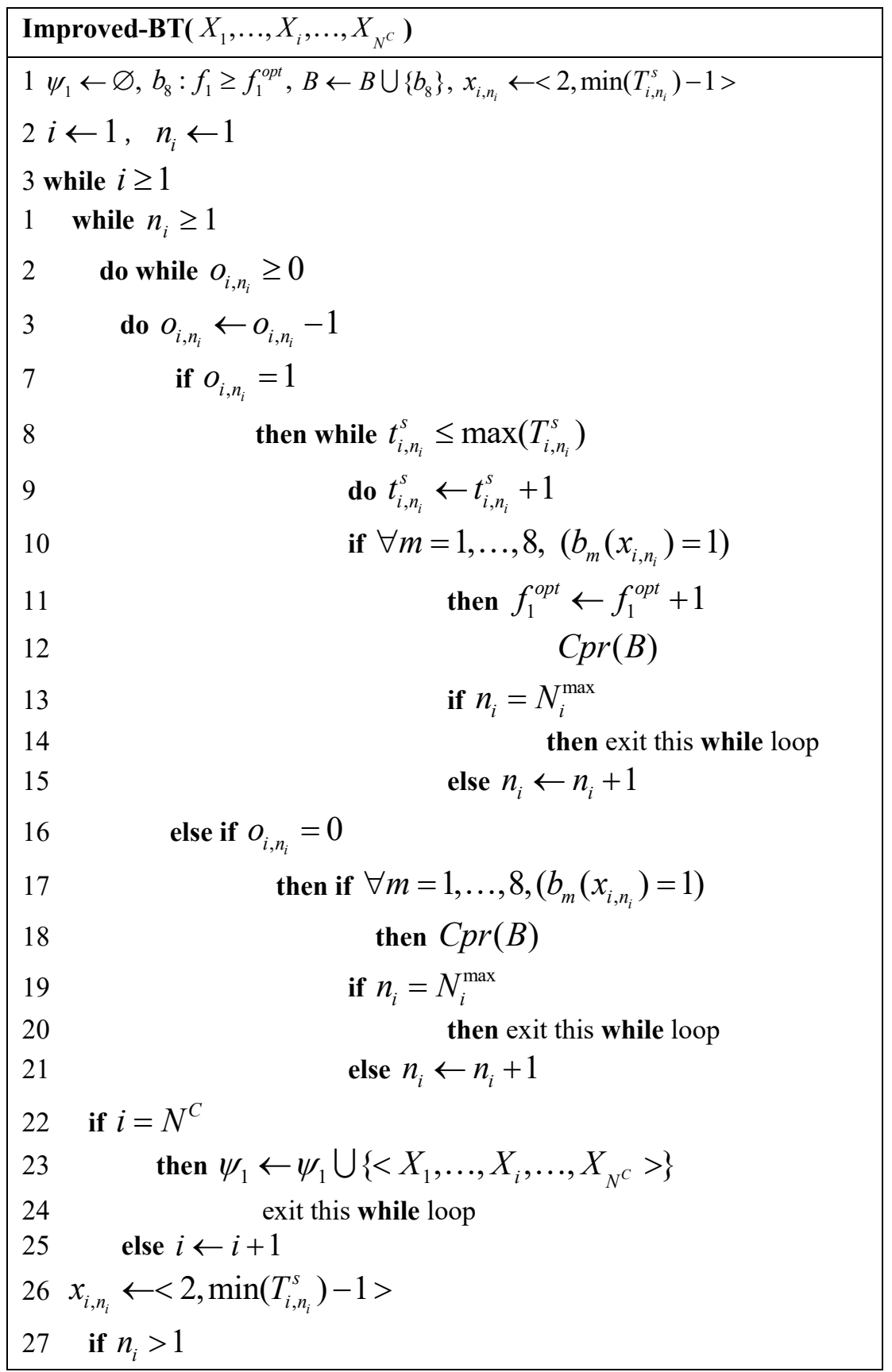


$28 \quad$ then $n_{i} \leftarrow n_{i}-1$

29 else $i \leftarrow i-1$ and $n_{i} \leftarrow N_{i-1}^{\max }$

30 return $\psi_{1}$

Based on the optimization solution of the upper layer model, the lower layer optimization model with the objective function (8) will be solved. The constraint $b_{8}$ in Improved-BT must be changed to $f_{2} \leq f_{2}^{\text {opt }}$, and the initial maximum value of the objective function $f_{2}$ be $f_{2}^{o p t}=d_{N^{R}}$.

\section{Case study}

\subsection{Case solving}

There are 15 rolling stocks in Chengdu Metro Line 2 in China. The daily capacity of the maintenance crew team is 5 in the planning horizon from day 1 to day 365 . At least 10 trains must be provided to the main line every day. Assume that each train was put into use at day 0 , that is, the latest end time $t_{i, j}^{0}$ for each maintenance is 0 . Let the solving time of each layer model be less than 1 minute. Different kinds of maintenances are listed in Table 1.

Table 1: $\quad$ Different kinds of maintenances in Chengdu Metro Line 2.

\begin{tabular}{lcll}
\hline Type $\left(y_{j}\right)$ & priority $\left(p_{j}\right)$ & cycle $\left(T_{j}^{\min } \sim T_{j}^{\max }\right)$ & duration $\left(d_{j}\right)$ \\
\hline heavy & 6 & $10-12$ years & 30 days \\
unwheeling & 5 & $5-6$ years & 24 days \\
light & 4 & $1.25-1.5$ years & 8 days \\
monthly & 3 & $82-98$ days & 3 days \\
weekly & 2 & $12-18$ days & 6 hours \\
pantograph & 1 & $4-7$ d ays & 1 hour \\
\hline
\end{tabular}

There are 1575 maintenances, 4725 variables, about one million constraints, and one hundred million iterations in this case. The model is solved using the improved backtracking algorithm on a PC equipped with as Inter Core i3-2310M $2.1 \mathrm{GHz}$, DRAM 2G, Windows 7 and .NET Framework 4.5. The solving processes of the upper layer model with the objective function (7) and the lower model with the objective function (8) are shown in Figure 1. Finally, the results of the maintenance scheduling are shown in Table 2. 

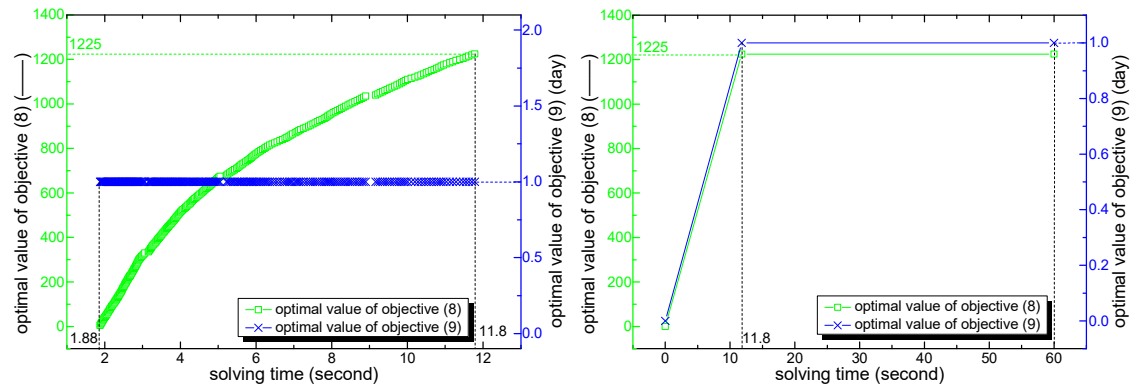

Figure 1: The solving processes of the upper model and the lower model.

Table 2: $\quad$ Scheduling results of the maintenance.

\begin{tabular}{ll}
\hline rolling stock $\quad$ maintenance type $($ start time $\sim$ end time) \\
\hline 1 & pantograph $(5 \sim 5,10 \sim 10, \cdots, 361 \sim 361)$ weekly $(13 \sim 13,30 \sim 30, \cdots, 364 \sim 364)$ \\
2 & pantograph $(5 \sim 5,10 \sim 10, \cdots, 361 \sim 361)$ weekly $(13 \sim 13,30 \sim 30, \cdots, 364 \sim 364)$ \\
3 & pantograph $(5 \sim 5,10 \sim 10, \cdots, 361 \sim 361)$ weekly $(13 \sim 13,30 \sim 30, \cdots, 364 \sim 364)$ \\
4 & pantograph $(5 \sim 5,10 \sim 10, \cdots, 361 \sim 361)$ weekly $(13 \sim 13,30 \sim 30, \cdots, 364 \sim 364)$ \\
5 & pantograph $(5 \sim 5,10 \sim 10, \cdots, 361 \sim 361)$ weekly $(13 \sim 13,30 \sim 30, \cdots, 364 \sim 364)$ \\
6 & pantograph $(6 \sim 6,11 \sim 11, \cdots, 362 \sim 362)$ weekly $(14 \sim 14,31 \sim 31, \cdots, 365 \sim 365)$ \\
7 & pantograph $(6 \sim 6,11 \sim 11, \cdots, 362 \sim 362)$ weekly $(14 \sim 14,31 \sim 31, \cdots, 365 \sim 365)$ \\
8 & pantograph $(6 \sim 6,11 \sim 11, \cdots, 362 \sim 362)$ weekly $(14 \sim 14,31 \sim 31, \cdots, 365 \sim 365)$ \\
9 & pantograph $(6 \sim 6,11 \sim 11, \cdots, 362 \sim 362)$ weekly $(14 \sim 14,31 \sim 31, \cdots, 365 \sim 365)$ \\
10 & pantograph $(6 \sim 6,11 \sim 11, \cdots, 362 \sim 362)$ weekly $(14 \sim 14,31 \sim 31, \cdots, 365 \sim 365)$ \\
11 & pantograph $(7 \sim 7,12 \sim 12, \cdots, 363 \sim 363)$ weekly $(15 \sim 15,33 \sim 33, \cdots, 353 \sim 353)$ \\
12 & pantograph $(7 \sim 7,12 \sim 12, \cdots, 363 \sim 363)$ weekly $(15 \sim 15,33 \sim 33, \cdots, 353 \sim 353)$ \\
13 & pantograph $(7 \sim 7,12 \sim 12, \cdots, 363 \sim 363)$ weekly $(15 \sim 15,33 \sim 33, \cdots, 353 \sim 353)$ \\
14 & pantograph $(7 \sim 7,12 \sim 12, \cdots, 363 \sim 363)$ weekly $(15 \sim 15,33 \sim 33, \cdots, 353 \sim 353)$ \\
15 & pantograph $(7 \sim 7,12 \sim 12, \cdots, 363 \sim 363)$ weekly $(15 \sim 15,33 \sim 33, \cdots, 353 \sim 353)$ \\
\hline
\end{tabular}

\subsection{Algorithm comparisons}

To illustrate the efficiency of Improved-BT algorithm, the BT1 algorithm with the static variable ordering heuristics according to the priority of the maintenance, the BT2 algorithm with the static variable ordering heuristics according to the car number ranking from small to big, the BT3 algorithm with the dynamic value ordering heuristics according to the maximum value of the start time, and the BT4 algorithm without constraint propagation technique are used to solve the upper layer model with the objective function (7). The process of each algorithm is shown in Figure 2. 

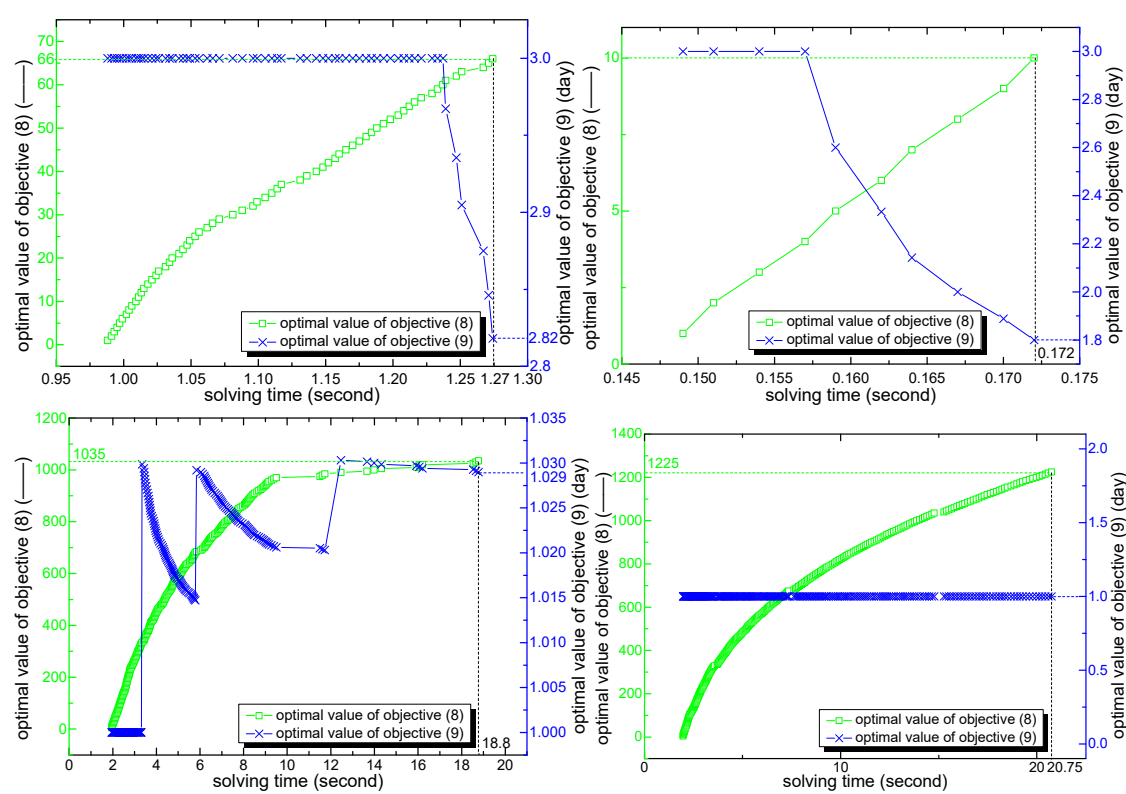

Figure 2: $\quad$ The solution processes of BT1, BT2, BT3 and BT4.

Figure 2 can verify that the Improved-BT algorithm is more high-quality and high-efficiency than other algorithms. Particularly, because of the constraint propagation technique, the iterations number of Improved-BT algorithm is 3992, while the iterations number of BT4 algorithm is 44529.

\section{Conclusion and future work}

In this paper, a multi-objective mixed integer nonlinear bi-level programming model for RSMS was established. Then an improved backtracking algorithm with two kinds of ordering heuristics and constraint propagation technique was designed to solve the model efficiently. This study can improve the efficiency of the rolling stock maintenance and can protect the safety of the train operation in the main line. Based on the proposed optimized model and algorithm, our future work is to study the integrated optimization problem of operation scheduling and maintenance scheduling to improve the service level of rolling stock bases and the mainline in the metro.

\section{References}

[1] Wu, H.J.S., Design and Development of Subway Trains MIS for Maintenance, Chengdu: Southwest Jiaotong University, 2012.

[2] Zeng, X.Y., Design of Maintenance Information System for Subway Rolling stock, Chengdu: Southwest Jiaotong University, 2012. 
[3] Tang, D.S., Design and Implementation of Metro Rolling stock Maintenance Scheduling Management System, Guangzhou: South China University of Technology, 2013.

[4] Andrés, J., Cadarso, L., and Ángel Marín, Maintenance scheduling in rolling stock circulations in rapid transit networks. Transportation Research Procedia, 10, 524-533, 2015.

[5] Peng, F., \& Ouyang, Y., Track maintenance production team scheduling in railroad networks. Transportation Research Part B Methodological, 46(10), 1474-1488, 2012.

[6] Liu, S.M., Theory and application of multi objective programming, Shanghai : Shanghai Jiaotong University Press, 2014.

[7] $\mathrm{Hu}, \mathrm{C} . Y$., Bi-level programming theory and its application in management, Beijing: Intellectual Property Publishing House, 2012.

[8] Golomb, S.B., Backtrack programming. ACM, 12, pp. 516-524, 1965.

[9] Bacchus, F. \& Vanrun, P., Dynamic variable ordering in CSPs. In Proceedings of the First International Conference on Principles and Practice of Constraint Programming, Cassis, France, pp. 258-275, 1995.

[10] Huang, J. \& Darwiche, A., A structure-based variable ordering heuristic for SAT. In Proceedings of the Eighteenth International Joint Conference on Artificial Intelligence, Acapulco, Mexico, pp. 1167-1172, 2003.

[11] Hooker, J., Logic, Optimization and Constraint Programming. Informs Journal On Computing, 14(4), pp. 295-321, 2002.

[12] Roossi, F., Van, B. P. \& Walsh, T., Handbook of Constraint Programming. Pisa: Elsevier, pp. 103-109, 2006. 